
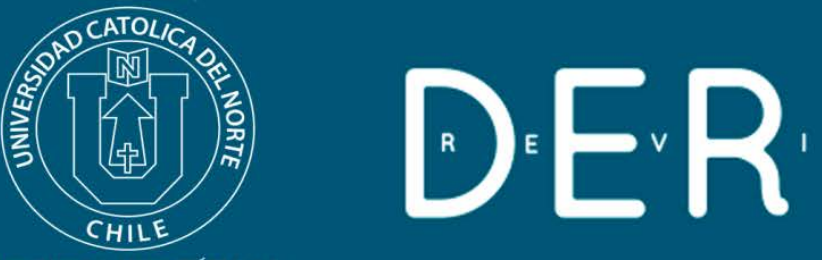

do) $10.22199 /$ issn.0718-9753-2021-0018

REVISTAS CIENTIIFICAS

de la Universidad Católica del Nort.
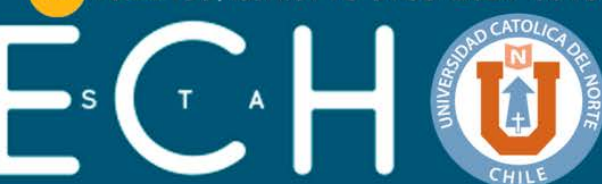

C o q u i m bo

ISSN: 0718-9753 (En línea)

\title{
Compensación y derechos punitivos en el Derecho Internacional: comentario a la sentencia de la Corte Internacional de Justicia en "Compensación debida por la República de Nicaragua a la República de Costa Rica"
}

\section{Compensation and punitive rights in International Law: Commentary on the Judgment of the International Court of Justice on "Compensation Owed by the Republic of Nicaragua to the Republic of Costa Rica"}

\author{
María Elisa Zavala Achurra' ${ }^{10}$ https://orcid.org/0000-0002-0202-2604
}

${ }^{1}$ Pontificia Universidad Católica de Chile, Santiago, Chile. Abogada. Magister en Derecho, U. de Harvard.@mezavala@uc.cl

(cc) BY

\section{Resumen:}

El reciente fallo de compensación dictado por la Corte Internacional de Justicia en el caso disputado entre Costa Rica y Nicaragua levanta puntos relevantes sobre las reparaciones en la esfera internacional. Asentado en doctrina y jurisprudencia que las compensaciones otorgadas en derecho internacional buscan reparar un daño causado, pero jamás castigar al estado infractor, resulta necesario analizar si en este caso aquel paradigma comienza a cambiar. Específicamente, la forma en que razonan los jueces de la mayoría, y algunas Declaraciones y una Opinión Separada al fallo, muestra que en este caso probablemente se otorgaron daños punitivos de facto. Incluso aunque aquello no hubiera ocurrido, surgen voces dentro de la misma Corte Internacional de Justicia que llaman a incluir los daños punitivos en este ordenamiento jurídico. En este contexto, el estudio de la concepción, rol y función de la compensación en el derecho internacional resulta sumamente relevante. Este comentario alerta sobre los desafíos que tribunales internacionales otorgaran daños punitivos.

Palabras Clave: Tribunales internacionales; Reparación; Daños punitivos.

\begin{abstract}
:
The recent compensation award issued by the International Court of Justice in the case that had Costa Rica and Nicaragua litigating raises relevant questions in relation to reparations in international law. It is established in doctrine and in jurisprudence that compensations that are awarded in international law seek to repair a damage that was caused, but never punish the damaging state. However, it is important to analyze whether that paradigm has started to change. Specifically, the way in which the Court reasoned, and some Declarations to it and one Separate Opinion, show that punitive damages were probably de facto awarded in this case. Even if that were not the case, voices inside the same International Court of Justice support the inclusion of punitive damages in this legal system. In this context, the study of the concept, role and function of compensation in international law is of the outmost relevance. This comment alerts about the challenges that would exist if international tribunals award punitive damages. Keywords: International court; Reparation; Punitive damages.
\end{abstract}




\section{Introducción}

El 2 de febrero de 2018, la Corte Internacional de Justicia pronunció tres fallos resolviendo casos relacionados que tenían a los vecinos Costa Rica y Nicaragua por partes. Una de aquellas sentencias, la que se analiza en el presente trabajo, es un fallo de compensación que estableció que Nicaragua debía compensar a Costa Rica por los daños que le causaron a este último estado las actividades ilegales en las que había incurrido el primero. El monto de la compensación que recibió Costa Rica por medio de este fallo fue objeto de discusiones entre los miembros de la Corte Internacional de Justicia, como se desprende de algunas Declaraciones y una Opinión Separada a la sentencia. Precisamente aquellas discusiones podrían implicar que la función por la cual se otorgó parte de la compensación a Costa Rica no fue reparatoria.

El presente comentario se centra en la sentencia de compensación referida, presentando un breve resumen de los aspectos relevantes de aquella decisión para este trabajo. Luego, se analiza el rol que la compensación juega en el Derecho Internacional. Si bien hasta el momento la jurisprudencia y doctrina internacional han entendido que los daños punitivos no forman parte de este sistema, el objeto del presente análisis es determinar si aquellos podrían regularse y otorgarse en este ordenamiento. En línea con esto, se busca también alertar de los desafíos que implicaría hacerlo. El examen que se presenta toma las Opiniones Separadas y Disidentes a la sentencia como punto de partida. Además, como punto de comparación y referencia, se realizan algunos contrastes con el derecho civil continental, específicamente con el ordenamiento chileno; y también con el common law, sobre todo con el desarrollo de los daños punitivos en Estados Unidos. En ambos sistemas, y como veremos también en el derecho internacional, la regulación de la indemnización, compensación, los roles que aquellas cumplen, y el potencial lugar que puedan ocupar los daños punitivos se encuentra actualmente aún en desarrollo. Los desafíos que esto planea son múltiples.

\section{Caso "Ciertas actividades llevadas a cabo por Nicaragua en la zo- na fronteriza" y "Construcción de un camino en Costa Rica a lo largo del Río San Juan"}

\subsection{Hechos del caso}

El 2 de febrero de 2018 la Corte Internacional de Justicia pronunció su falló en el caso "Compensación debida por la República de Nicaragua a la República de Costa Rica" (International Court of Justice, 2018). Esta sentencia es la secuela de aquella dictada en el año 2015 por la misma Corte Internacional de Justicia, donde se estableció la 
obligación que tenía Nicaragua de compensar a Costa Rica por ciertas actividades ilegales en las que había incurrido (Corte Internacional de Justicia, 2019).

A modo de contexto, es relevante tener en cuenta que estos dos países vecinos tienen como frontera natural el Río San Juan, de gran importancia económica y natural para ambos, el uso del cual se encuentra en parte regulado en tratados suscritos entre las partes, como el Tratado de 1858 (International Court of Justice, 2009). Ambos estados utilizan el río en cuestión, y han realizado actividades en las cercanías al mismo.

El caso del año 2015 se remonta al procedimiento que inició Costa Rica en el año 2010, en contra de Nicaragua. La demanda, en primer lugar, imputa una violación a la soberanía del estado demandante, que habría ocurrido por una supuesta incursión, ocupación y uso, por Nicaragua, específicamente por su ejército, de territorio costarricense (International Court of Justice, 2015, p. 668). Aquellas infracciones habrían tenido lugar en el contexto de la construcción de un caño a través de Costa Rica, desde el Río San Juan hacia la Laguna los Portillos, y además por ciertas actividades de dragado y limpiado que se realizaron en aquél mismo río. Estas infracciones también se habrían cometido en la zona denominada "territorio disputado", área previamente definida por la misma Corte (ver, International Court of Justice, 2011; International Court of Justice, 2013b). En segundo término, se le imputaron a Nicaragua violaciones al derecho internacional medioambiental, en las que habría incurrido por realizar actividades que causaron daño en el territorio de Costa Rica (International Court of Justice, 2015, p. 668). Actividades que, por lo demás, habrían violado las obligaciones procedimentales que rigen en materia internacional medioambiental, como la de notificar y consultar a los terceros estados potencialmente afectados, o la de realizar estudios de impacto medioambiental antes de implementar proyectos riesgosos. En tercer lugar, también se imputaron a Nicaragua violaciones a obligaciones convencionales, específicamente a aquellas contenidas en el Tratado de 1858 (International Court of Justice, 2015, p. 668). Como consecuencia de las infracciones en que habría incurrido Nicaragua, Costa Rica demandó que se le repare por el daño sufrido, dentro de lo cual solicitó una partida de reparación que consista en compensación para remediar los daños materiales sufridos.

Posteriormente, en el año 2011, Nicaragua inició un procedimiento en contra de Costa Rica, imputándole también violaciones al derecho internacional medio ambiental. En primer término, infracciones a las obligaciones medioambientales aplicables, contenidas tanto en la costumbre internacional como en tratados aplicables, en términos similares a las imputaciones previamente realizadas por Costa Rica contra Nicaragua (International Court of Justice, 2015, p. 669). En segundo lugar, Nicaragua alegó incumplimientos de obligaciones sustantivas de derecho internacional medioambiental (International Court of Justice, 2015, p. 669). Éstas se habrían cometido por el estado demandado al realizar trabajos de construcción de caminos cerca al 
límite de ambos países en la zona del Río San Juan. En tercer término, Nicaragua alega violaciones a su soberanía e integridad territorial, específicamente en la zona de aquél mismo río. Finalmente, Nicaragua también demandó reparaciones por las infracciones cometidas en su contra.

Es importante considerar que la Corte ordenó medidas provisionales para ambos Estados en el año 2011 (International Court of Justice, 2011). Luego, en el año 2013, la Corte Internacional de Justicia decidió juntar ambos procedimientos en uno, para mayor celeridad y economía procesal (International Court of Justice, 2013). Poco después, la Corte decidió mantener vigentes las Medidas Provisionales que se habían ordenado en contra de los estados, e incluso impuso algunas adicionales (International Court of Justice, 2013b).

\subsection{Sentencia de fondo}

La sentencia que resolvió el fondo de ambos casos se dictó en el año 2015. Respecto de las imputaciones realizadas por Costa Rica, en resumen, la Corte Internacional de Justicia estableció que: (i) Nicaragua había violado la soberanía de Costa Rica por medio de la presencia de miembros de su ejército en el territorio disputado (International Court of Justice, 2015, pár. 99); (ii) que Nicaragua no violó obligaciones procedimentales de derecho internacional medioambiental (International Court of Justice, 2015, pár. 105, 108, 109, 112); (iii) que Nicaragua tampoco violó obligaciones sustantivas de derecho internacional medioambiental (International Court of Justice, 2015, pár. 120); (iv) que Nicaragua incumplió las medidas provisionales que la Corte Internacional de Justicia le había impuesto (International Court of Justice, 2015, pár. 129, 136); y (v) que Nicaragua sí violó los derechos de navegación que tenía bajo el Tratado de 1858 (este tema se discutió en extenso en un caso previo entre estos mismos países, que la Corte resolvió con anterioridad. Ver, para más detalles International Court of Justice, 2009). Finalmente, y en lo que es relevante para este comentario, se determinó que Costa Rica tiene derecho a recibir compensación económica por las violaciones en que Nicaragua incurrió, que el mismo fallo establece, pero sólo respecto de aquellas que le causaron un daño material estimable (International Court of Justice, 2015, pár. 142). Respecto del resto de las infracciones, sin embargo, a la Corte Internacional de Justicia le parece suficiente la declaración que la misma Corte hace de aquellas como forma de reparación (International Court of Justice, 2015, pár. 139).

Por su parte, respecto de las alegaciones de Nicaragua, la Corte determinó que: (i) Costa Rica había incumplido obligaciones procedimentales de derecho internacional medioambiental, específicamente (a) no realizar un estudio de impacto medioambiental antes de comenzar la construcción de los caminos antes referidos (International Court of Justice, 2015, pár. 156, 159, 162); sin embargo, (b) determinó que no se habían incumplido las obligaciones convencionales relacionadas con lo anterior, porque la Convención de Diversidad Biológica no contiene una obligación de 
realizar un estudio de aquél tipo antes de realizar proyectos que puedan ser riesgosos para el medioambiente (International Court of Justice, 2015, pár. 164); y (c) que tampoco violó obligaciones de consulta que fueran aplicables a este caso (International Court of Justice, 2015, pár. 171-173); (ii) en relación con las obligaciones sustantivas de esta misma materia, contenidas en la costumbre internacional, todas las alegaciones de Nicaragua se descartan por no haberse probado que al estado demandante se le había causado un daño transfronterizo significativo por las actividades del estado demandado (International Court of Justice, 2015, pár. 196, 207, 213, $216,217) ;($ iii) en relación con las obligaciones medioambientales sustantivas contenidas en tratados internacionales, tampoco Nicaragua logró probar daños que harían a Costa Rica responsable de las violaciones que el primero le imputó (International Court of Justice, 2015, pár. 220); y (iv) se rechazaron también las supuestas infracciones a la soberanía y territorio de Nicaragua (International Court of Justice, 2015, pár. 220). En relación con la compensación solicitada por Nicaragua, la Corte explica que, como la única infracción de Costa Rica que se tuvo por acreditada fue la de no realizar un estudio de impacto medio ambiental, no procede compensación, ya que la forma de reparación adecuada para remediar aquella violación es la satisfacción (International Court of Justice, 2015, pár. 225-226).

\subsection{Sentencia de Compensación}

En la sentencia de fondo, la Corte sugiere a las partes llegar a un acuerdo sobre el monto de la compensación que Nicaragua le debe a Costa Rica, a falta del cual se podrá iniciar un nuevo procedimiento para determinar el quantum de aquella compensación:

The relevant material damage and the amount of compensation may be assessed by the Court only in separate proceedings. The Court is of the opinion that the Parties should engage in negotiation in order to reach an agreement on these issues. However, if they fail to reach such an agreement within 12 months of the date of the present Judgment, the Court will, at the request of either Party, determine the amount of compensation on the basis of further written pleadings limited to this issue. (International Court of Justice, 2015, pár. 142) ${ }^{1}$

Esto último fue efectivamente lo que ocurrió, y en el año 2017 Costa Rica informó a la Corte Internacional de Justicia que no fue posible alcanzar un acuerdo, y se inició el procedimiento de compensación (International Court of Justice, 2018, pár. 11-12).

\footnotetext{
1 “El daño material relevante y el monto de compensación puede ser determinado por la Corte sólo en un procedimiento separado. La Corte es de la opinión que las Partes deberían comenzar negociaciones con miras a alcanzar un acuerdo en estos temas. Sin embargo, si no logran alcanzar un acuerdo dentro de 12 meses a partir de la fecha de la presente Sentencia, la Corte determinará, a petición de cualquiera de las Partes, el monto de compensación sobre la base de nuevos memoriales que se limiten a este tema". NdT: La presente y todas las traducciones son libres del original en inglés, realizadas de la autora.
} 
Como explica la sentencia analizada, Costa Rica solicitó compensación por dos categorías de daños: (i) daños medioambientales (esta es la primera vez que la Corte Internacional de Justicia conoce un caso por compensación de daños de este tipo); y (ii) los costos y gastos incurridos por aquél estado como consecuencia de las actividades ilegales de Nicaragua (International Court of Justice, 2018, pár. 36). Para determinar el monto que Nicaragua debe indemnizar por aquellos conceptos, las partes propusieron distintas metodologías, y la Corte, en vez de adoptar una de ellas, tomó partes de una y otra, y junto con otros elementos adicionales llegó a un mecanismo que le pareció idóneo (International Court of Justice, 2018, pár. 52).

Finalmente, la Corte da por establecidos daños por concepto de afectaciones al medio ambiente consistentes en deterioros o pérdidas ascendentes a US\$120,000 (International Court of Justice, 2018, pár. 86), y por concepto de restauración que se eleva a US\$2,708.39 (International Court of Justice, 2018, pár. 87). Es muy importante remarcar que justo antes de dar por establecidos el primero de aquellos daños, explica lo siguiente:

The Court recalls, as outlined in paragraph 35 above, that the absence of certainty as to the extent of damage does not necessarily preclude it from awarding an amount that it considers approximately to reflect the value of the impairment or loss of environmental goods and services. In this case, the Court, while retaining some of the elements of the 'corrected analysis', considers it reasonable that, for the purposes of its overall valuation, an adjustment be made to the total amount in the 'corrected analysis' to account for the shortcomings identified in the preceding paragraph. (International Court of Justice, 2018, pár. 86)².

Además, la sentencia comentada reconoce que se han probado daños por concepto de costos y gastos que son consecuencia directa de las acciones ilegales de Nicaragua que alcanzan los US\$236.032,16 (International Court of Justice, 2018, pár. 147).

Existen interesantes Opiniones Separadas y Declaraciones que realizan ciertos ministros de la Corte al fallo comentado. Las que son atingentes al análisis que aquí se realiza se tratarán en la sección 3.

\footnotetext{
2 "La Corte recuerda, como se adelantó en el párrafo 35 arriba, que la ausencia de certeza respecto de la extensión del daño no necesariamente le impide adjudicar un monto que considere que refleja aproximadamente el valor del deterioro o pérdida de bienes o servicios medioambientales. En este caso, la Corte, mientras conserva algunos de los elementos del 'análisis corregido', considera que es razonable, para el propósito de su evaluación global, realizar un ajuste al monto total en el 'análisis corregido' para dar cuenta de defectos identificados en el párrafo precedente".
} 


\section{La compensación en los distintos sistemas jurídicos Amnistías fis- cales: concepto y dos ejemplos chilenos}

\subsection{Compensación y derecho civil}

En términos generales, se entiende que los fines de una pena (en este caso una monetaria) son múltiples: busca reparar un daño sufrido, disuadir a otros infractores de cometer las mismas, y castigar al criminal. En los derechos de tradición civil continental, y como ocurre por ejemplo en Chile, la función principal de la pena civil es la reparación por medio de la indemnización de perjuicios. De esta forma, el enfoque no está en el castigo al autor del daño, ni en el reproche de culpabilidad que este pudiera merecer (Barros Bourie, 2006, p. 256).

En línea con lo anterior, la máxima transversal a la tradición jurídica del derecho civil continental, sintetizada en los términos de Cabrillac (2010), es la siguiente: "...debe repararse todo el daño, pero nada más que el daño" (p. 163). Ese mismo principio se recoge en los códigos de los países que siguen una tradición de derecho civil. En Chile, el principio de reparación integral encuentra su base legal en el artículo 2329 del Código Civil, que, si bien se encuentra dentro de la regulación del daño extracontractual, aplica también en materia contractual (Barros Bourie, 2006, p. 255). El mismo principio se reconoce ampliamente en la jurisprudencia de nuestros tribunales superiores (Hernán Gutiérrez San Martín con Municipalidad de Viña del Mar, 2018, cons. undécimo). De la misma forma, y desde antiguo, se reconoce en doctrina que la reparación por daños sufridos debe ser completa, lo que, como explica Alessandri Rodríguez (1943), tiene como consecuencia que (i) "El monto de la reparación depende de la extensión del daño [...] (ii) El monto de la reparación no puede ser superior ni inferior al daño" (p. 545).

De esta forma, no es posible otorgar daños punitivos en sistemas de derecho civil, ya que, como se explica en detalle en la siguiente sección, aquellos constituyen reparaciones que un infractor debe pagar por sobre el monto del daño efectivamente causado a la víctima. Los daños punitivos son tal punto ajeno a la tradición civil continental que autores chilenos han incluso planteado la inconstitucionalidad de otorgar daños de este tipo en Chile (Corral Talciani, 2003, p. 68). A pesar de esto, existen algunos países que tradicionalmente han seguido una tradición de derecho civil, como España, Francia y Argentina, quienes han incorporado a sus sistemas jurídicos normas que desafían este entendimiento (García-Matamoros y Arévalo-Ramírez, 2019 , p. 184). Esto evidencia que la regulación de las indemnizaciones sigue en desarrollo en estos países, cosa que también ocurre en el common law. La siguiente sección se encarga de esa tradición jurídica. 


\subsection{Compensación y common law}

En la tradición del common law la compensación puede jugar un rol distinto al que juega en los sistemas legales de tradición civil. Específicamente, en ciertos casos las cortes pueden ordenar el pago de daños "extracompensatorios" (Sharkey, 2003, p. 363), como lo son los daños punitivos, ejemplares, o vindicativos (conceptos que en el presente trabajo se utilizan de forma indistinta). Entender estos es necesario para determinar el efecto y alcance que puede tener el fallo de la Corte Internacional de Justicia analizada en el presente comentario.

Estos daños pueden definirse como "...damages awarded as punishment for the defendant's acts rather than as compensation to make a plaintiff whole..." (Yarn, 1999)3. Es posible encontrar una definición similar a aquella en el Restatement (Second) of Torts "Punitive damages are damages, other than compensatory or nominal damages, awarded against a person to punish him for his outrageous conduct and to deter him and others like him from similar conduct in the future"4 (American Law Institute, 1979, § 908). Existen diferencias en la manera en que están regulados estos daños en los distintos países que los reconocen (ver Gotanda, 2007, p. 190). El foco de esta sección estará en el trato que reciben aquellos daños en Estados Unidos, dada la vasta doctrina y jurisprudencia que se ha referido a ellos. Además, los grandes montos que se han litigado y otorgado en materia de daños punitivos han ido en constante aumento durante las últimas décadas (Sunstein, Hastie, Payne, Schkade, y Viscusi, 2002, p. 1), lo que ha llamado aún más la atención jurídica y mediática hacia este tipo de daños.

En términos generales, los daños ejemplares se otorgan de forma adicional a los que buscan reparar daños, y tienen fines de prevención y de disuasión de los ilícitos que se sancionan (Polinsky y Shavell, 2000, p. 765; ver, para más detalles, Sunstein, Kahneman y Schkade, 1998). Así, las principales justificaciones de los daños ejemplares son el castigo (o retribución) y la disuasión (Sharkey, 2003, pp. 356-357). La Corte Suprema de Estados Unidos ha sostenido de forma repetida que los daños punitivos sirven esas dos funciones, las cuales se encuentran relacionadas (Allen, 2004, p. 8), aquel tribunal explicó que:

...compensatory and punitive damages, although usually awarded at the same time by the same decisionmaker, serve different purposes [...] Compensatory damages 'are intended to redress the concrete loss that the plaintiff has suffered by reason of the defendant's wrongful conduct' [...] By contrast, punitive damages serve a broader function; they are aimed at

\footnotetext{
${ }^{3 \prime}$... daños que se otorgan como un castigo por los actos del demandado y no como una compensación para reparar al demandante".

4 "Daños punitivos son daños, distintos a los compensatorios o nominales, que se otorgan contra una persona para castigarlo por su conducta reprochable, y para disuadirlo a él y a otros de realizar conductas similares en el futuro".
} 
deterrence and retribution ${ }^{5}$. (State Farm Mut. Automobile Ins. Co. v. Campbell, 2003, p. 416)

De todas formas, la justificación que prevalece es la primera de ellas, es decir, la retribución del daño causado a la víctima (Sharkey, 2003, p. 359). La segunda justificación, sin embargo, ha sido defendida por aquellos académicos que siguen la corriente de law and economics. Ellos explican que una disuasión ideal o perfecta requiere que el infractor internalice todos los costos causados por su acción (ver Landes y Posner, 1987; Shavell, 1987). Sin embargo, en algunas circunstancias aquello no ocurre, como cuando alguien ha cometido un acto que daña a muchas personas, y sabe que un porcentaje de ellas no lo demandará. En esos casos, sancionar a la víctima con daños ejemplares asegura la disuasión de la infracción cometida.

Si se contrastan los daños punitivos con aquellos que se permite otorgar en los sistemas de derecho civil, se detecta que los primeros tienen una doble función: reparar el daño causado a la víctima, y evitar un daño futuro a la sociedad en general (a través de la disuasión) (García-Matamoros y Arévalo-Ramírez, 2019, p. 186). Sin embargo, en la tradición civil, los daños sólo buscan una reparación a la víctima, y no se consideran efectos presentes o futuros a la sociedad en general. Esta diferencia es muy relevante, y tiene implicancias que han de considerarse cuando se examinan los daños punitivos en el contexto internacional.

\subsection{El camino que tomó el derecho internacional}

El Derecho Internacional Público ha seguido mayoritariamente al derecho civil continental en materia de compensaciones económicas. El principio de reparación integral ('full reparation' en inglés, o 'restituto ad integrum' en su formulación latina) tiene amplia acogida en este sistema jurídico. Así por ejemplo lo estableció, hace bastante tiempo, el fallo de la Corte Permanente de Justicia en el caso de la Fábrica de Chórzow (The Factory at Chorzow (Claim for Indemnity) (The Merits). Germany v. Poland, 1928), sentencia a la que el fallo comentado refiere a modo de introducción de la compensación que luego procede a otorgar (International Court of Justice, 2018, pár. 29). Por su parte, Crawford (2013), explica que el principio básico en materia de reparaciones es aquel de acuerdo al cual debe remediarse todo daño sufrido, buscando eliminar las consecuencias de los ilícitos cometidos de la mayor forma posible, y que por eso mismo los Artículos sobre Responsabilidad de los Estados utilizan el término 'full reparation' (pp. 480-482; además véase International Law Commission, 2008, art. 34).

\footnotetext{
5 “...daños punitivos y compensatorios, aunque suelen otorgarse al mismo tiempo y por el mismo juzgador, sirven distintos propósitos [...]. Los daños compensatorios buscan reparar la pérdida concreta que el demandando ha sufrido por culpa de la conducta ilegal cometida por el demandado. [...] En cambio, los daños punitivos cumplen una función más amplia; ellos están orientados a la disuasión y a la retribución".
} 
No sólo es lógico que los daños punitivos no se condigan con el sistema de compensación del Derecho Internacional, sino que además existe amplio consenso que los daños ejemplares no forman parte ni se pueden otorgar en este sistema. En primer término, la Comisión de Derecho Internacional discutió la procedencia de estos daños al redactar los Artículos sobre Responsabilidad de los Estados, y en los comentarios a aquellos explica, de forma muy clara, que los daños punitivos no pueden otorgarse en Derecho Internacional:

In other words, the function of article 36 is purely compensatory, as its title indicates. Compensation corresponds to the financially assessable damage suffered by the injured State or its nationals. It is not concerned to punish the responsible State, nor does compensation have an expressive or exemplary character ${ }^{6}$. (International Law Commission, 2008, art. 36, pár. 4)

Sin embargo, debe recordarse que una versión anterior de estos artículos, específicamente el borrador de 1996, contenía un artículo 42 a partir del cual podría interpretarse que los daños punitivos sí formaban parte del Derecho Internacional, en algunos casos (Tams, 2002, p. 1167). Específicamente, el borrador establecía que podrían otorgarse daños punitivos frente a violaciones graves, para que la pena reflejara la seriedad del incumplimiento (Draft articles on state responsibility with commentaries thereto adopted by the international law commission on first reading, 1997, art. 42). La redacción actual de los artículos referidos eliminó aquella referencia, e incluso el comentario de la Comisión de Derecho Internacional a los mismos expresamente explica que los daños punitivos no proceden ni aún frente a violaciones graves de Derecho Internacional (International Law Commission, 2008, cap. III, pár. 5)

En materia de tratados internacionales, destaca el tratamiento que la Convención Americana de Derechos Humanos le da a las compensaciones. El artículo 63 de dicho instrumento básicamente reproduce el estándar fijado por la Corte Permanente de Justicia en el ya mencionado caso de la Fábrica de Chorzow (The Factory at Chorzow (Claim for Indemnity) (The Merits). Germany v. Poland, 1928):

Cuando decida que hubo violación de un derecho o libertad protegidos en esta Convención, la Corte dispondrá que se garantice al lesionado en el goce de su derecho o libertad conculcados. Dispondrá asimismo, si ello fuera procedente, que se reparen las consecuencias de la medida o situación que ha configurado la vulneración de esos derechos y el pago de una justa indemnización a la parte lesionada. (Convención Americana sobre Derechos Humanos, 1978, art. 63, no. 1)

\footnotetext{
6 "En otras palabras, la función del artículo 36 es puramente compensatoria, como su título lo indica. La compensación corresponde a los daños financieramente estimables que han sufrido un Estado afectado o sus nacionales. No tiene que ver con un castigo al Estado responsable, ni tiene un carácter expresivo o ejemplar".
} 
Por su parte, y como se adelantó, la jurisprudencia internacional también ha reafirmado que los daños punitivos no forman parte del derecho internacional. Existen autores que incluso afirman que ningún tribunal o corte internacional contemporánea ha otorgado daños punitivos (Wittich, 2010, p. 671). La Corte Interamericana de Derecho Humanos así lo estableció en las Reparaciones y Costas de Velásquez Rodríguez Vs. Honduras (1989, pár. 316), al negar lugar a compensaciones ejemplarizantes que los solicitantes buscaban conseguir (García-Matamoros y Arévalo-Ramírez, 2019, p. 202). Lo mismo se ha fallado por aquella misma corte en casos posteriores (Godínez Cruz Vs. Honduras, 1989; Garrido y Baigorria Vs. Argentina, 1998). Dicho tribunal, sin embargo, se alejó de esa posición en el caso Myrna Mack Chang Vs. Guatemala (2003), al ordenar daños que consideraban la gravedad de las violaciones causadas por Guatemala (van Aaken, A. y Motoc, 2018, p. 240). La Opinión Separada del entonces juez de la Corte Interamericana -hoy juez de la Corte internacional de JusticiaCançado Trindade expresamente defendió la necesidad de otorgar daños punitivos (Myrna Mack Chang Vs. Guatemala, 2003).

Por su parte, la Corte Europea de Derechos Humanos también ha descartado la procedencia de daños punitivos en su jurisdicción en Varnava and Others v. Turkey (2009, pár. 223). En el mismo sentido se pronunció la Comisión Internacional ChileEstados Unidos que se refirió a los daños relativos al caso del asesinato del senador Orlando Letelier en Estados Unidos (Orrego Vicuña, 1992).

En materia de arbitraje internacional ocurre algo especial porque en algunas circunstancias se han otorgado daños punitivos, aunque aquello ha sido infrecuente (Gotanda, 2009, p. 555). De todas formas, existen autores que argumentan que es esperable que los fallos arbitrales internacionales comiencen a otorgar daños punitivos con mayor frecuencia en el futuro (Petsche, 2013, pp. 31-32). Si aquello ocurre, resultará interesante seguir el desarrollo que aquellos daños tengan en la esfera internacional.

Finalmente, en lo que se refiere a la jurisprudencia internacional, y para despejar toda duda, la Corte Internacional de Justicia tuvo la oportunidad de referirse a este tema por primera vez en la sentencia comentada, y descarta de forma clara la posibilidad de otorgar daños punitivos en su sede (International Court of Justice, 2018, pár. 31).

La doctrina también suele estar conteste en que los daños punitivos no tienen cabida en el Derecho Internacional. Así lo explica, por ejemplo, Crawford (2013, p. 524). De todas formas, es relevante tener en cuenta que existe una parte menor de la doctrina que sostiene que los derechos punitivos deben reconocerse en el Derecho Internacional, y explican cómo ciertos fallos de hecho han otorgado daños punitivos.

Dentro de los disidentes más citados en esta materia se encuentra Jorgensen (1997), quien, si bien reconoce que en el Derecho Internacional Público contemporá- 
neo no se admiten los daños punitivos, explica que ellos, en la práctica, se han otorgado por tribunales internacionales. Explica que en el caso Laura M. B. Janes et al. (U.S.A.) v. United Mexican States (Nielsen, 1925), por ejemplo, a Estados Unidos se le adjudicó una suma de dinero, que debía pagar México, país en el que habían asesinado a un ciudadano americano, crimen que México no castigó (Jorgensen, 1997, pp. 248-249). La comisión explicó que, si bien México no era directamente responsable por el crimen, si tenía responsabilidad para con Estados Unidos (y no con el reclamante) de una forma punitiva (Nielsen, 1925, p. 89). Tras aquel análisis la autora concluye que: "...the idea that pecuniary reparation may exceed the limits of restitution and in some instances go beyond even moral damages and amount to punishment would seem to be a general principle of law, and it may be that practice is simply lagging behind principle" (Jorgensen, 1997, p. 266) ${ }^{7}$. Sin embargo, existen autores como Tams (2002, p. 1167) y Wittich (2010, p. 671) que, analizando esos mismos casos, descartan que de ellos se pueda concluir que establecieron daños punitivos.

Finalmente, lo que está en juego si se otorgan daños ejemplares sin que estos estén incorporados al Derecho Internacional, es decir, sin una fuente que los reconozcan, es la soberanía de los estados. De hecho, Oppenheim (1928, pár. 156), en las primeras versiones de su conocido tratado, explicaba que los daños punitivos efectivamente conllevaban ese riesgo.

\section{Daños punitivos de facto en el fallo analizado}

Es claro que el fallo analizado no establece ni otorga de forma directa daños punitivos. De hecho, excluye expresamente esa posibilidad, como se indicó. Sin embargo, el análisis que continúa busca entender si acaso en los hechos esta sentencia otorgó daños punitivos, o si al menos otorgó daños que no correspondían propiamente a una forma de compensación que sigue el principio de reparación integral.

Como se adelantó, las opiniones separadas y declaraciones de algunos de los jueces de la Corte resultan interesantes para el tema en comento. Ellas dejan entrever que dentro de la Corte Internacional de Justicia debe haber existido una discusión interesante sobre la forma en que se otorgan las compensaciones en esa sede, y quizás incluso sobre los daños punitivos.

En primer término, llama la atención la Declaración del Juez Gevorgian, quien alerta sobre el método de determinación de perjuicios que utilizó la Corte. La sentencia comentada utiliza un análisis global para determinar la cantidad de perjuicios que Nicaragua debe pagar a Costa Rica como consecuencia de los daños medio am-

\footnotetext{
7 “...la idea que la reparación pecuniaria pueda exceder los límites de la restitución y en algunos casos ir incluso más allá que los daños morales y significar un castigo parece ser un principio general del derecho, y podría ser que la práctica simplemente se ha quedado atrás de aquel principio".
} 
bientales causados (International Court of Justice, 2018, pár. 78), lo que quiere decir que no determina los daños de forma separada para cada una de las partidas solicitadas. Esta Declaración explica que utilizar un análisis como aquél, que no distinga montos de compensación por cada concepto, conlleva el riesgo de que se otorguen de facto daños punitivos a un estado (Gevorgian, 2018, pár. 3). Esto se suma a su preocupación sobre ciertas partidas de daños respecto de las cuales, a su parecer, Costa Rica no alcanzó la carga de la prueba que le correspondía para darlos por establecidos (Gevorgian, 2018, pár. 4-8).

En segundo término, la Jueza Donoghue, quien suscribió una Opinión Separada al fallo, también razona de forma similar al juez anteriormente referido. Esta Jueza disidió en parte con la mayoría de la Corte, específicamente en lo relativo a los daños medioambientales que Costa Rica solicitó como consecuencia de la restauración que iba a ser necesaria para remediar los daños sufridos en el medioambiente (Donoghue, 2018). En su Opinión separada explica que respecto de aquella partida de daños, ella era del parecer que Costa Rica no fue capaz de probar que ha incurrido o que ciertamente incurrirá en aquellos gastos a futuro (Donoghue, 2018, pár. 33-35). Adicionalmente, explica que, si bien concurrió con la mayoría de la Corte Internacional de Justicia en los demás daños medio ambientales otorgados, es de la idea que Costa Rica no alcanzó la carga de la prueba que le correspondía (Donoghue, 2018, pár. $4,5,13$ y 19). Dado que en Derecho Internacional rige el principio que cada parte debe probar los hechos que alega, y sin existir en este caso alguna situación que requiriera revertir la aplicación de aquel principio, Costa Rica se encontraba en la obligación de acreditar todos los daños que solicitó, y no le parece que así haya ocurrido (Donoghue, 2018, pár. 4, 5, 13 y 19). Finalmente, explica que si bien concuerda con la Corte en que la valoración de daños medioambientales 'puros' inevitablemente requiere que se realice una aproximación, eso no puede autorizar una justificación débil de los montos que se otorgan (Donoghue, 2018, pár. 32). Del análisis de esta Opinión Separada se desprende que esta Jueza también tenía reparos respecto de la forma en que se otorgaron y valoraron los daños en la sentencia comentada, y aunque no se dice de forma explícita, otorgar compensaciones por daños no acreditados puede tornarse en un castigo para el infractor que no es permitida en el Derecho Internacional.

Finalmente, para el análisis que se realiza también es interesante la Declaración del Juez Bhandari, quien se muestra favorable al fallo de la mayoría. Respecto de la determinación de daños medioambientales este juez está de acuerdo con la Corte en que algunas veces es difícil determinarlos, y por eso mismo sugiere que se recurra a consideraciones de equidad para llegar al monto deseado (Bhandari, 2018, pár. 12). Además, si bien reconoce que en el Derecho Internacional Público no es posible otorgar daños punitivos, sugiere que se analice la procedencia de los mismos en materia medioambiental (Bhandari, 2018, pár. 17). Fundándose en doctrina y legislación doméstica, especialmente de India, su país de origen, explica que sería admisible 
otorgar daños punitivos en esta materia, dada la gravedad de las infracciones medioambientales, y el daño a la humanidad que causan (Bhandari, 2018, pár. 19). En suma, concluye que la posibilidad de otorgar daños por este concepto estaría justificada, en esta materia, por consideraciones humanitarias (Bhandari, 2018, pár. 21).

El análisis realizado es interesante ya que evidencia que probablemente el tema de los daños punitivos en sede internacional fue un tema discutido entre los miembros de la Corte Internacional de Justicia. Lo que llama más la atención es la declaración del Juez Bhandari, quien va más lejos que otros autores y fallos en la materia, argumentando expresamente que los daños punitivos tendrían o deberían tener cabida en Derecho Internacional, en abierta contradicción con la visión de la Comisión de Derecho Internacional, como se vio. Recordemos además que el Juez Cançado Trindade también defendió la necesidad de incorporar daños punitivos al ordenamiento internacional cuando era juez de la Corte Interamericana, como se mencionó.

\section{Mirando al futuro}

Si bien actualmente el Derecho Internacional Público no permite otorgar daños punitivos, no es difícil imaginar que aquello pueda ocurrir en el futuro. Si ellos fueran a incluirse en este ordenamiento, sería relevante que quienes participen en la redacción de las normas estudien los desafíos que los daños punitivos presentan en los países que los contemplan. La comunidad académica, por su parte, puede también alertar sobre aquellos, y proponer formas de superarlos en la esfera internacional. Si uno estudia el tratamiento de los daños punitivos en Estados Unidos se detectan algunos de los siguientes problemas, los cuales también existirían si este tipo de daños se otorgaran por tribunales internacionales.

Un primer problema es determinar el monto de los daños punitivos que se han de otorgar. En comparación a la compensación que busca reparar un daño causado, en materia de daños punitivos, dado que ellos constituyen una sanción al infractor, no existe un monto exacto ni forma determinada de probar cuál es el monto de daños punitivos que se debe otorgar. Si bien algunos académicos han creado fórmulas para determinar aquello (ver Polinsky y Shavell, 1998), no existe un método universalmente aceptado para lograrlo. En derecho internacional, esto implicaría un desafío para los jueces que tengan que determinar la procedencia y montos de los daños punitivos que se pudieran otorgar.

Un segundo problema, íntimamente relacionado con el anterior, es que los daños punitivos pueden ser excesivos, o puede castigarse al infractor más de una vez por los mismos hechos. Que estos daños sean una sanción no implica que ellos puedan ascender a cualquier monto. En Estados Unidos se ha estudiado este fenómeno desde distintas perspectivas. Algunos explican que los daños excesivos pueden ser 
causados por un cálculo inadecuado de los daños que se otorgan. Huang (2014, p. 2020) explica que en aquellos casos que se otorgan daños punitivos por haberse dañado a varias personas, la forma de multiplicar los daños puede ser errónea toda vez que los costos fijos del daño causado se multipliquen de la misma forma que aqueIlos costos que son variables. Otros se han enfocado en los problemas que causan las demandas consecutivas por daños punitivos, lo que supone un desafío porque la primera sentencia por daños punitivos no impide que otros afectados por la infracción puedan también iniciar procesos por los mismos hechos (Colby, 2003, p. 587). Por ejemplo, en el conocidísimo caso Roginsky v. Richardson-Merrell, Inc. (1967), el Juez Friendly explicó que "We know of no principle whereby the first punitive award exhausts all claims for punitive damages and would thus preclude future judgments..." (p. 839) ${ }^{8}$. Existen otros casos donde los daños otorgados son increíblemente altos aún cuando los hechos no han afectado a muchas personas (Sharkey, 2003, p. 352). Todas estas dificultades pueden también existir de otorgarse daños punitivos por tribunales internacionales. Un daño transfronterizo, por ejemplo, puede fácilmente afectar a más de un estado. En esos casos, los mismos problemas que se han estudiado en los países que otorgan daños punitivos existirían en materia internacional. Este desafío es aún mayor en un sistema jurídico integrado por varios tribunales internacionales, algunos con jurisdicción general y otros especializada, que no se encuentran claramente jerarquizados (ver Charney, 1998; Kingsbury, 1999; Lowe, 2000).

En tercer lugar, es usual que al otorgarse daños punitivos se tenga en cuenta la capacidad económica del infractor. La lógica es que, si el fin de los daños punitivos es, en parte, disuadir infracciones futuras, entonces debe atenderse al costo relativo que la sanción tiene para el infractor (Levit, 1974, p. 484). Esto no es un problema especialmente grave en Estados Unidos, pero de todas formas presenta desafíos para los tribunales. En materia internacional, en cambio, determinar los daños en atención a la capacidad económica de los países sí parece un desafío mayor dado que en la esfera internacional se intenta no hacer diferencias entre estados en atención al dinero que ellos tengan.

Un cuarto problema se refiere a los límites dentro de los cuáles se otorgan estos daños. Más que un problema, en realidad los límites son una respuesta a los problemas anteriormente mencionados (Huang, 2014, p. 1032). En el ámbito internacional, sería necesario establecer cuáles son aquellos límites, los cuáles no será simple exportar desde jurisdicciones nacionales. Lo anterior ya que, en Estados Unidos, por ejemplo, algunos de esos límites encuentran su origen en cláusulas constitucionales, mientras que otras se han desarrollado de forma local y por tanto varían de estado a estado (García-Matamoros y Arévalo-Ramírez, 2019, p. 208).

\footnotetext{
8 "No conocemos ningún principio de acuerdo al cual la primera sentencia que otorga daños punitivos agota todas las acciones de daños punitivos, prohibiendo sentencias futuras..."
} 
Finalmente, los daños punitivos pueden además poner en jaque la certeza jurídica. Este problema es en parte causado por las dificultades previamente referidas, e implica una falta de predictibilidad producida por la posibilidad de que se otorguen daños punitivos a un infractor (ver Gotanda, 2009). Dado que la predictibilidad es necesaria para el funcionamiento de un ordenamiento jurídico, la incertidumbre que genera (i) la posibilidad de ser sancionado con daños ejemplares; y (ii) el monto al cual aquellos pueden ascender afecta la legitimidad de un ordenamiento jurídico. A la vez, aquello supone un desafío para los tribunales, quienes tienen que decidir cuándo procede otorgar estos daños, y por qué montos. Y para los estados es difícil saber cómo conducirse en sus relaciones internacionales si no saben cómo o con qué sanciones serán castigados si infringen las normas que los rigen.

\section{Conclusiones}

Del análisis del reciente fallo sobre compensaciones de la Corte Internacional de Justicia es posible establecer, con bastante claridad, que en el Derecho Internacional Público contemporáneo no es posible otorgar daños punitivos. De hecho, la misma Corte tuvo la oportunidad de declarar aquello en el fallo comentado. Sin embargo, al mismo tiempo se advierte que hay corrientes doctrinarias que apoyan la introducción de estos daños en este sistema jurídico, y que explican que sí existe jurisprudencia internacional que, al menos de facto, ha otorgado daños punitivos. En este grupo ya se incluía el Juez Cançado Trindade, y se suma ahora el Juez Bhandari, quien explícitamente llama a estudiar la necesidad de introducir daños ejemplares en Derecho Internacional, y argumenta que los mismos estarían permitidos, por ejemplo, en materia medioambiental, por consideraciones humanitarias.

Siendo aquél el estado del arte sobre esta materia es posible advertir dos implicancias relacionadas con la introducción de los daños punitivos a este sistema jurídico. En primer término, la Corte Internacional de Justicia debe ser cuidadosa en no otorgar daños punitivos de facto. Como se ha explicado, existe bastante consenso doctrinario respecto a que la teoría del derecho de las compensaciones y del derecho de la responsabilidad de los estados no contempla daños punitivos. Sin embargo, los autores que defienden la existencia de aquellos efectivamente argumentan que, en la práctica, se han otorgado este tipo de daños en la esfera internacional. Por lo mismo, los estados y la comunidad académica deben estar atentos a atisbos de daños ejemplares que puedan aparecer en fallos que se dicten. Como se dijo, lo que está en juego si se otorgan daños punitivos sin una fuente legal que lo autorice es la soberanía misma de los estados. En segundo término, si lo que se quiere es otorgar daños punitivos, $u$ otorgarlos en determinadas materias, en la línea de lo que propone el Juez Bhandari, lo que se debiera hacer es introducir regulación expresa al respecto. Si bien estos daños no están permitidos, sí sería posible que se regularan como lex specialis para alguna materia en particular. Si aquello ocurre, sería recomendable que 
quienes participen en la redacción de aquellas normas revisen el desarrollo de la doctrina y jurisprudencia de daños punitivos en los países en que aquellos existen. El estudio de ellos en Estados Unidos es particularmente abundante, y puede ilustrar este proceso.

\section{Referencias Bibliográficas}

van Aaken, A. y Motoc, I. (Eds.). (2018). The European Convention on Human Rights and general international law. Oxford: Oxford University Press. doi: 10.1093/oso/9780198830009.001.0001

Alessandri Rodríguez, A. (1943). De la responsabilidad extracontractual en el derecho civil chileno (título 35 del libro IV del Código civil). Santiago: Universitaria.

Allen, M. P. (2004). The Supreme court, punitive damages and state sovereignty. George Mason law review, 13(1), 1-68. Recuperado de https://bit.ly/3xTUov6

American Law Institute. (1979). Restatement of the Law second, Torts (Vol. 4). Saint Paul, MN, A.L.I.

Bhandari, D. (2018). Separate opinion of Judge Bhandari. Certain activities carried out by Nicaragua in the border area (Costa Rica v. Nicaragua). Compensation owed by the Republic of Nicaragua to the Republic of Costa Rica. Judgment of 2 february 2018. Reports of judgments, advisory opinions and orders (International Court of Justice), (1133), 96-104. Recuperado de https://bit.ly/3jM6wdN

Barros Bourie, E. (2006). Tratado de responsabilidad extracontractual. Santiago: Jurídica de Chile.

Cabrillac, R. (2010). Droit des obligations (9a ed.). Paris: Dalloz.

Charney, J. I. (1998). Is international law threatened by multiple international tribunals? (Vol. 271, Recucil des cours). Boston, MA: Martinus Nijhoff. doi: 10.1163/18758096_pplrdc_A9789041112101_03

Colby, T. B. (2003). Beyond the multiple punishment problem: punitive damages as punishment for individual, private wrongs. Minnesota law review, 87(3), 583-678. Recuperado de https://bit.ly/3k13npq

Convención Americana sobre Derechos Humanos (Pacto de San José). Organización de los Estados Americanos, San José, Costa Rica, 18 de julio de 1978. Recuperado de https://bit.ly/3ogz53i

Corral Talciani, H. (2003). Lecciones de responsabilidad civil extracontractual. Santiago: Jurídica de Chile. 
Compensación y derechos punitivos en el Derecho Internacional...

Crawford, J. (2013). State responsibility: the general part. Cambridge: Cambridge University Press. doi: 10.1017/CBO9781139033060

Donoghue, J. E. (2018). Separate opinion of Judge Donoghue. Certain activities carried out by Nicaragua in the border area (Costa Rica v. Nicaragua). Compensation owed by the Republic of Nicaragua to the Republic of Costa Rica. Judgment of 2 february 2018. Reports of judgments, advisory opinions and orders (International Court of Justice), (1133), 85-95. Recuperado de https://bit.ly/3yO6SVq

Draft articles on state responsibility with commentaries thereto adopted by the international law commission on first reading, 97-02583 (International Law Commission enero de 1997). Recuperado de https://bit.ly/3BJmNX5

The Factory at Chorzow (Claim for Indemnity) (The Merits). Germany v. Poland, File E. c. XIII Docket XIV: I (Permanent Court of International Justice, 13 de septiembre de 1928). Recuperado de https://bit.ly/2VgKoyM

García-Matamoros, L. V. y Arévalo-Ramírez, W. (2019). Desarrollos recientes sobre daños punitivos en el derecho continental, en el common law, en el Sistema Interamericano de Derechos Humanos y en el derecho internacional. Revista de derecho privado, 37(183), 183-220. doi: 10.18601/01234366.n37.08

Garrido y Baigorria Vs. Argentina (Reparaciones y Costas), Serie C, No 91 (Corte Interamericana de Derechos Humanos 27 de agosto de 1998). Recuperado de https://bit.ly/3841bbi

Gevorgian, K. (2018). Declaration of Judge Gevorgian. Certain activities carried out by Nicaragua in the border area (Costa Rica v. Nicaragua). Compensation owed by the Republic of Nicaragua to the Republic of Costa Rica. Judgment of 2 february 2018. Reports of judgments, advisory opinions and orders (International Court of Justice), (1133), 105-108. Recuperado de https://bit.ly/3h3C25a

Godínez Cruz Vs. Honduras (Reparaciones y Costas), Serie C No. 8 (Corte Interamericana de Derechos Humanos 21 de julio de 1989). Recuperado de https://bit.ly/3mqiTOq

Gotanda, J. Y. (2007). Damages in private international law (Vol. 326, Recucil des cours). Boston, MA: Martinus Nijhoff. doi: 10.1163/18758096_pplrdc_A9789004166165_02

Gotanda, J. Y. (2009). The unpredictability paradox: punitive damages and interest in international arbitration. The journal of world investment \& trade, 10(4), 553-572. doi: $10.1163 / 221190009 \times 00303$ 
Hernán Gutiérrez San Martín con Municipalidad de Viña del Mar, Rol N²811-2018 (Corte Suprema 28 de marzo de 2018). Recuperado de https://westlawchile.cl id: CL/JUR/1506/2018

Huang, B. I. (2014). Surprisingly punitive damages. Virginia law review, 100(5), 10271060. Recuperado de https://bit.ly/3BL34qc

International Court of Justice. (2009). Dispute regarding navigational and related rights (Costa Rica v. Nicaragua). Judgment of 13 july 2009. Reports of judgments, advisory opinions and orders (International Court of Justice), (959), 213-272. Recuperado de https://bit.ly/3BNqrPB

International Court of Justice. (2011). Certain activities carried out by Nicaragua in the border area (Costa Rica v. Nicaragua). Request for the indication of provisional measures. Order of 8 march 2011. Reports of Judgments, advisory opinions and orders (International Court of Justice), (1013), 6-28. Recuperado de https://bit.ly/38G1Bos

International Court of Justice. (2013). Certain activities carried out by Nicaragua in the border area (Costa Rica v. Nicaragua). Joinder of proceedings. Order of 17 april 2013. Reports of Judgments, advisory opinions and orders (International Court of Justice), (1043), 166-171. Recuperado de https://bit.ly/38L4R1K

International Court of Justice. (2013b). Certain activities carried out by Nicaragua in the border area (Costa Rica v. Nicaragua). Construction of a road in Costa Rica along the San Juan River (Nicaragua v. Costa Rica). Request presented by Costa Rica for the indication of new provisional measures. Order of 22 november 2013. Reports of Judgments, advisory opinions and orders (International Court of Justice), (1051), 354370. Recuperado de https://bit.ly/3yXX2AN

International Court of Justice. (2015). Certain activities carried out by Nicaragua in the border area (Costa Rica v. Nicaragua) and construction of a road in Costa Rica along the San Juan River (Nicaragua v. Costa Rica). Judgment of 16 december 2015. Reports of judgments, advisory opinions and orders (International Court of Justice), (1088), 665741. Recuperado de https://bit.ly/38Frc0S

International Court of Justice. (2018). Certain activities carried out by Nicaragua in the border area (Costa Rica v. Nicaragua). Compensation owed by the Republic of Nicaragua to the Republic of Costa Rica. Judgment of 2 february 2018. Reports of judgments, advisory opinions and orders (International Court of Justice), (1133). Recuperado de https://bit.ly/3yN6h6s

Jorgensen, N. (1997). A reappraisal of punitive damages in international law. British yearbook of international law, 68(1), 247-266. doi: 10.1093/bybil/68.1.247 
Compensación y derechos punitivos en el Derecho Internacional...

Kingsbury, B. (1999). Is the proliferation of international courts and tribunals a systemic problem? New York University Journal of international law \& politics, 31(4), 679696. Recuperado de https://bit.ly/3jXp6i2

Landes, W. M. y Posner, R. A. (1987). The economic structure of tort law. Cambridge, MA: Harvard University Press.

Levit, V. B. (1974). Punitive damages today. Insurance law journal, 1974(9), 483-487.

Lowe, V. (2000). Overlapping jurisdiction in international tribunals. The Australian year book of international law online, 20(1), 191-204. doi: 10.1163/26660229-020-01900000012

Nielsen, Fred K. (1925, Noviembre 16). Laura M. B. Janes et al. (U.S.A.) v. United Mexican States. Reports of international arbitral awards, 4, 82-98. Recuperado de https://bit.ly/3D1 mM2f

Oppenheim, L. (1928). International law (vol. 1). Londres: Longmans.

Orrego Vicuña, F. (1992, Febrero 11). Dispute concerning responsibility for the deaths of Letelier and Moffitt (United States, Chile). Reports of international arbitral awards, 25, 3-19. Recuperado de https://bit.ly/3D1iXtM

Petsche, M. (2013). Punitive damages in international commercial arbitration: a conflict of laws lesson. Journal of international arbitration, 30(1), 31-48. Recuperado de https://bit.ly/3ge2t82

Polinsky, M. y Shavell, S. (1998). Punitive damages and economic analysis. Harvard law review, 111(4), 868-962. doi: 10.2307/1342009

Polinsky, M. y Shavell, S. (2000). Punitive damages. En B. Bouckaert, and G. De Geest (Eds.), Encyclopedia of law and economics (Vol. 2, pp. 764-781). Cheltenham: Edward Elgar. Recuperado de https://bit.ly/2W7BR1m

Roginsky v. Richardson-Merrell, Inc., 378 F.2d 832 (United States Court of Appeals, Second Circuit 4 de abril 1967). Recuperado de https://bit.ly/37XRMSt

Sharkey, C. M. (2003). Punitive damages as societal damages. The Yale law journal, 113(2), 347-454. Recuperado de https://bit.ly/3AXiKWX

Shavell, S. (1987). Economic analysis of accident law. Cambridge, MA: Harvard University Press. Recuperado de https://bit.ly/3xXglth

State Farm Mut. Automobile Ins. Co. v. Campbell, 538 U.S. 408 (US Supreme Court 7 de Abril 2003). Recuperado de https://bit.ly/3h68jbZ 
Sunstein, C. R., Hastie, R., Payne, J. W., Schkade, D. A. y Viscusi, W. K. (2002). Punitive damages: how juries decide. Chicago, IL: The University of Chicago Press.

Sunstein, C., Kahneman, D. y Schkade, D. (1998). Assessing punitive damages (with notes on cognition and valuation in law). The Yale law journal, 107(7), 2071-2153. Recuperado de https://bit.ly/2XDKG3H

Tams, C. (2002). Do Serious Breaches Give Rise to Any Specific Obligations of the Responsible State? European journal of international law, 13(5), 1161-1180. Recuperado de https://bit.ly/3k8BknV

Varnava and Others v. Turkey, Applications nos. 16064/90, 16065/90, 16066/90, $16068 / 90,16069 / 90,16070 / 90,16071 / 90,16072 / 90$ and 16073/90 (European Court of Human Rights 18 de Septiembre de 2009). Recuperado de https://bit.ly/3ybfgOC

Velásquez Rodríguez Vs. Honduras (Reparaciones y Costas), Serie C No. 7 (Corte Interamericana de Derechos Humanos 21 de julio de 1989). Recuperado de https://bit.ly/3k6J1ek

Wittich, S. (2010). Punitive damages. En J. Crawford, A. Pellet, y S. Olleson (Eds.), The law of international responsibility (pp. 667-675). Oxford: Oxford University Press.

Yarn, D. H. (Ed.). (1999). Dictionary of conflict resolution. Hoboken, NJ: Wiley. Recuperado de https://bit.ly/3h55PL5

\section{Para citar este artículo bajo Norma APA 6a ed.}

Zavala Achurra, M. E. (2021). Compensación y derechos punitivos en

el Derecho Internacional: comentario a la sentencia de la Corte In-

ternacional de Justicia en "Compensación debida por la República

de Nicaragua a la República de Costa Rica". Revista de derecho (Co-

quimbo. En línea), 28, e3336. https://doi.org/10.22199/issn.0718-

9753-2021-0018 